\title{
Differential activation of frontal and parietal regions during visual word recognition: An optical topography study
}

\author{
Markus J. Hofmann, ${ }^{\mathrm{a}, *, 1}$ Martin J. Herrmann, ${ }^{\mathrm{b}, *, 1}$ Ippeita Dan, ${ }^{\mathrm{c}}$ Hellmuth Obrig, ${ }^{\mathrm{d}}$ \\ Markus Conrad, ${ }^{\mathrm{a}}$ Lars Kuchinke, ${ }^{\mathrm{a}}$ Arthur M. Jacobs, ${ }^{\mathrm{a}}$ and Andreas J. Fallgatter ${ }^{\mathrm{b}}$ \\ ${ }^{a}$ Department of Psychology, Free University Berlin, Germany \\ ${ }^{\mathrm{b}}$ Department of Psychiatry and Psychotherapy, University Hospital Wuerzburg, Germany \\ ${ }^{\mathrm{c}}$ National Food Research Institute, Tsukuba, Japan \\ ${ }^{\mathrm{d}}$ Department of Neurology, Charité, Berlin, Germany
}

Received 24 August 2007; revised 13 November 2007; accepted 18 December 2007

Available online 3 January 2008

\begin{abstract}
The present study examined cortical oxygenation changes during lexical decision on words and pseudowords using functional NearInfrared Spectroscopy (fNIRS). Focal hyperoxygenation as an indicator of functional activation was compared over three target areas over the left hemisphere. A 52-channel Hitachi ETG-4000 was used covering the superior frontal gyrus (SFG), the left inferior parietal gyrus (IPG) and the left inferior frontal gyrus (IFG). To allow for anatomical inference a recently developed probabilistic mapping method was used to determine the most likely anatomic locations of the changes in cortical activation [Tsuzuki, D., Jurcak, V., Singh, A.K., Okamoto, M., Watanabe, E., Dan, I., 2007. Virtual spatial registration of stand-alone fNIRS data to MNI space. NeuroImage 43 (4), 1506-1518.

Subjects made lexical decisions on 50 low and 50 high frequency words and 100 pseudowords. With respect to the lexicality effect, words elicited a larger focal hyperoxygenation in comparison to pseudowords in two regions identified as the SFG and left IPG. The SFG activation difference was interpreted to reflect decision-related mechanisms according to the Multiple Read-Out Model [Grainger, J., Jacobs, A.M., 1996. Orthographic processing in visual word recognition: A multiple read-out model. Psychological Review 103, 518-565]. The greater oxygenation response to words in the left IPG suggests that this region connects orthographic, phonological and semantic representations. A decrease of deoxygenated hemoglobin was observed to low frequency in comparison to high frequency words in a region identified as IFG. This region's sensitivity to word frequency suggests its involvement in
\end{abstract}

\footnotetext{
* Corresponding authors. M.J. Hofmann is to be contacted at Department of Psychology, Free University Berlin, Habelschwerdter Allee 45, 14195 Berlin, Germany. Fax: +493083855620 . M.J. Herrmann, Clinic for Psychiatry and Psychotherapy, University of Wuerzburg, Fuechsleinstr. 15, 97080 Wuerzburg, Germany. Fax: +49 93120177550.

E-mail address: mhof@zedat.fu-berlin.de (M.J. Hofmann).

${ }^{1}$ The correspondence authors contributed equally to this work.

Available online on ScienceDirect (www.sciencedirect.com).
}

grapheme-phoneme conversion, or its role during the selection of preactivated semantic candidates.

(C) 2007 Elsevier Inc. All rights reserved.

Keywords: fNIRS; Optical imaging; Lexical decision; Word frequency; Lexicality; Probabilistic mapping

\section{Introduction}

The lexical decision task is a well established paradigm in psycholinguistics (Jacobs and Grainger, 1994). When subjects decide whether a string of letters represents a word or not (pseudoword, PW) two robust effects have been established. Readers react faster to words than to PWs. This was termed the lexicality effect. The other effect within the word-category is demonstrated when reaction times (RTs) to high frequency words are compared to RTs to low frequency words. This word frequency effect indicates a faster processing of common words compared to uncommon words and is the probably most robust finding in the word recognition literature (Jacobs and Grainger, 1994).

These behavioral findings were investigated by neuroimaging to identify the different brain areas involved in the task.

The key areas for the lexicality effect are the superior frontal gyrus (SFG) including the medial and middle frontal gyrus, and the left inferior parietal gyrus (IPG) including the angular and supramarginal gyrus. Both regions showed a greater response to words than to PWs during a feature detection task in a PET study (see Price, 2000, Fig. 10, second row, reanalysis of Brunswick et al., 1999). Also fMRI studies revealed a larger BOLD contrast in SFG and IPG for words compared to PWs. This was demonstrated in visual lexical decision (Binder et al., 2003; Kuchinke et al., 2005), silent articulation and phonological lexical decision tasks (Ischebeck et al., 2004). The latter study made subjects decide on whether or not a presented letter string sounds like a word if read aloud. 
Functional-anatomical findings can be interpreted in the light of the Multiple Read-Out Model (MROM, Grainger and Jacobs, 1996; Jacobs et al., 1998, 2003), a computational model of word recognition. Briefly, intralexical decision criteria of lexical activation generate a "yes"-response to word stimuli while an extralexical temporal threshold mechanism generates a "no"-response to PW stimuli. Fiebach et al. (2007) suggested that the putative role of the SFG lies in executive and control functions. More specifically the $\mathrm{SFG}$, an area involved in decision-related processes, can be assumed to respond differently as a function of which of the two response mechanisms postulated by the MROM is active: Thus it can be postulated that the "yes" and the "no" reaction will elicit different neural responses in decision-related brain regions such as the SFG (cf. Price, 2000, p. 353). Generally in line with this model, Ischebeck et al. (2004) argued that SFG activation reflects control functions with regard to retrieval of semantic information from posterior areas (for a review, see Binder et al., 1999). Thus words containing semantic information, elicit larger activations than PWs because the latter are devoid of semantic content.

The IPG, particularly the angular gyrus, has an even longer history as a target area in higher order language processing especially at the hinge between reading, writing and overt language production. More than a century ago Dejerine (1891) associated lesions in the IPG with a syndrome later termed "alexia with agraphia". His patient developed an inability to read and write after an IPG-lesion and concluded that IPG is critical for the 'memory' of the visual word form. Later Geschwind (1965) observed that IPG-lesioned patients are not able to understand words when they are spelt. Therefore he concluded that "It is a region which turns written language into spoken language and vice versa" (Geschwind, 1965, p. 278; cf. Damasio and Damasio, 1983).

Beyond lesion studies the analysis of developmental disorders of reading and writing point at the role of the IPG as a pivot between orthographic and phonological representations. Pugh et al. (2000) provided evidence that dyslexia can be conceived as a disorder of relating print to sound and vice versa, which corresponds to a disruption of the projections between the IPG, and occipital as well as temporal cortical areas (Booth et al., 2004; Horwitz et al., 1998; but see Kronbichler et al., 2006).

To sum up IPG can be conceived as a hub mediating the transfer between reading, writing, overt language production and semantic processing. Clinically, Price (2000) has pointed out that patients with an IPG lesion not only show impairments in reading and writing, but also perform poorly on semantic tasks (Hart and Gordon, 1990).

With respect to the word frequency effect the left inferior frontal gyrus (IFG) plays a major role since it is more activated in response to low than to high frequency words. An early PET study revealed a non-significant trend using a naming task (Fiez et al., 1999). Consecutive fMRI research rendered the word frequency effect the probably best-replicated finding of fMRI studies on word recognition. Larger BOLD contrasts in the IFG for low freqency words were reported in a silent articulation (Ischebeck et al., 2004; Kronbichler et al., 2004), a visual (Fiebach et al., 2002, 2003; Carreiras et al., 2006), an auditory (Prabhakaran et al., 2006) and a phonological lexical decision task (Carreiras et al., 2006; Ischebeck et al., 2004; Nakic et al., 2006; Prabhakaran et al., 2006).

The finding of a lesser activation for high frequency words was interpreted according to the dual route model (Coltheart et al., 2001; Fiebach et al., 2002). This model assumes that in low frequency words the so-called assembled route generates a phonological representation applying grapheme-phoneme correspondence rules: For each grapheme the corresponding phoneme is retrieved to generate a phonological representation. On the contrary high frequency words will be mainly processed by the addressed route generating a phonological representation by matching the whole word to the phonological representation. Fiebach et al. (2002) suggested that the predominance of generating a phonological representation by computing grapheme-phoneme correspondences for low frequency words elicits the greater IFG activation.

The present study introduces functional near-infrared spectroscopy (fNIRS) into the field of word recognition, a method assessing changes in cortical oxygenation by applying near-infrared light to measure changes in tissue attenuation. The relative transparency of biological tissue to light in the near infrared spectrum allows for optical tissuespectroscopy in a depth of some centimeters. Applied on the intact skull light-attenuation changes can thus be assessed in the cerebral cortex. Since oxygenated and deoxygenated hemoglobin have differential absorption spectra (i.e. "colors"), focal cortical hyperoxygenation can be reliably detected. The physiological basis of this measure of cortical activity is the fact that an increase in regional cerebral blood flow (rCBF) is closely coupled both spatially and temporally to neuronal activity. This so-termed neurovascular coupling is the basis of all modern imaging techniques such as BOLD-contrast $\mathrm{AMRI}$ and positron emission tomography (PET, Villringer and Dirnagl, 1995).

Thus fNIRS results are physiologically comparable to fMRI and PET results. However, its spatial resolution is rather coarse (Obrig and Villringer, 2003). Beyond this shortcoming fNIRS combines a number of features extremely attractive for language research. Being compatible with a natural environment and silent, the method's advantage has been proven in a number of previous studies in language research even in earliest infanthood (Fallgatter et al., 1998; Herrmann et al., 2006; Homae et al., 2006; Horovitz and Gore, 2004; Noguchi et al., 2002; Pena et al., 2003; Taga et al., 2003; Wartenburger et al., 2007, Watanabe et al., 1998). Here we challenge the methodology's potential to explore its versatility and reliability to differentiate activation in the three target areas discussed above (SFG/IPG/IFG). To frame the challenge in a more than descriptive way, we apply a recently developed procedure (Tsuzuki et al., 2007), which projects topographical data based on skull landmarks (e.g. 10-20-system) into a 3D reference frame (MNI-space, Montreal Neurological Institute). Though the resulting MNI-coordinates are subject to inter-individual error the procedure allows for a probabilistic reference to cortical areas on the brain's surface.

Our study is motivated by the perspective to elucidate the neuronal correlates of word processing not only in adult healthy volunteers, but also to extend the research to patients with neuropsychological deficits, and to link imaging results to developmental studies, by allowing to readily examine the emergence of literacy in children. To our knowledge this is the first fNIRS study attempting to disentangle the functional specificity of two neighboring areas, i.e. the SFG and the IFG. For this purpose, it was necessary to use the probabilistic mapping method.

\section{Methods \\ Participants}

Twelve right-handed healthy native German speaking subjects participated in the experiment ( 6 female, mean age 26 , ranging from 22 to 30). They were neurologically healthy and did not suffer from any language or speech impairment. Subjects were seated in a comfortable chair in a dimly lit room. The distance from eyes to monitor was about $50 \mathrm{~cm}$. 


\section{Materials}

The 200 experimental stimuli comprised 100 words and 100 PWs. All stimuli were bisyllabic and consisted of 4 to 7 letters. The number of letters was cross-balanced between words and PWs. The $100 \mathrm{PW}$ stimuli were pronounceable and were generated by stringing together legal syllables (taken from Hofmann et al., 2007).

The words used included 50 low frequency and 50 high frequency nouns. Mean word frequency was $2 /$ million ( $S D: 1$ ) for low frequency words (e.g. "Reling" [railing], "Sichel" [sickle]), and 229/ million (SD: 141) for high frequency words (e.g. Vater [father], Sache [matter], Baayen et al., 1995). Word frequency differed significantly $(t=11.4, \mathrm{p}<0.001)$. The number of letters and number of orthographic neighbors was balanced across categories. The frequency of the highest frequent neighbor did not differ $(t=0.1)$. Type and token mean bigram and letter frequencies were taken from the lemma database of Hofmann et al. (2007) and did not differ across cells $(t$ 's $\leq 0.1)$.

To assure that all low frequency words were known to a native German speaker, they were tested in a pre-experiment (10 subjects). These participants did not participate in the main experiment and were instructed to mark words that were not well known to them. This led to the replacement of four words from the initial stimulus list.

\section{Experimental procedure}

Participants were instructed to decide whether or not a presented letter string was a meaningful word and to respond by pressing one of two buttons using the index of the respective hand. Since motor responses may contaminate the cerebral activations of interest half of the participants were instructed to press the left button for words and the right button for PWs, and the other half responded vice versa. Accuracy was emphasized over speed.

The 200 experimental trials were presented in two blocks each containing 100 trials, preceded by 10 practice trials. Stimuli were presented in a pseudo-randomized fashion. Maximally three words or PWs were allowed to be presented consecutively.

At the beginning of each trial a fixation cross (“+”) was presented. After a randomly varied interval of $500-1000$ ms the stimulus was presented in white uppercase letters on a black background until a response was given. Then five hash marks (“\#\#\#\#\#”) were presented for $3500 \mathrm{~ms}$, followed by a blank screen for $500 \mathrm{~ms}$. There was no feedback on the response.

\section{Data acquisition}

Stimulus presentation and behavioral data acquisition relied on Presentation Software (Windows XP). Stimuli were presented on a 17 inch monitor with a screen refresh rate of $70 \mathrm{~Hz}$.

Cerebral oxygenation changes were sampled at $10 \mathrm{~Hz}$ by a Hitachi optical topograph (ETG-4000, Hitachi Medical. Co., Kashiwa, Japan). The system is a continuous wave device which measures changes in attenuation at 2 wavelengths $(695$ and $830 \mathrm{~nm}$, $\pm 20 \mathrm{~nm}$ ) and hence allows for the differentiation of two dynamic absorbers ([oxy-Hb] and [deoxy-Hb]). Lock-in technique is used to differentiate between wavelengths. Equipped with 16 light emitting and 17 detector probes 52 channels can be measured quasisimultaneously. Concentration changes in [oxy-Hb] and [deoxy-Hb] were calculated based on a modified Beer-Lambert approach (Cope and Delpy, 1988).
Inter-optode distance was $3 \mathrm{~cm}$. The array of 52 measurement positions (yellow circles Fig. 1) covered an area of $\sim 6 \times 30 \mathrm{~cm}$. As is illustrated in Fig. 1 the probe array was positioned on the subject's head with the medial detector of the lowest optode row corresponding to T3 of the 10-20 system while the lower edge of the probe set was fixed $1 \mathrm{~cm}$ above the inion (red circles in Fig. 1). For the definition of the 10-20 system (Jasper, 1958) the onsets of the zygomatic bones were defined as preauricular points (cf. Jurcak et al., 2007, Fig. 2D).

\section{Data analysis}

For outlier correction of the behavioral data, each RT deviating more than 2 standard deviations from the subject's mean was excluded from further behavioral analysis.

Event related oxygenation changes were analyzed by means of the General Linear Model (GLM), as proposed by Schroeter et al. (2004). To correct for artifacts due to heartbeat, data were low-pass filtered at $0.6 \mathrm{~Hz}$. For each pairwise comparison, a three predictor model was used. The first pairwise comparison was conducted to assess the lexicality effect. The prediction terms consisted of words, PWs, and behavioral errors, respectively. The second pairwise comparison was conducted to assess the word frequency effect. The prediction terms were, low frequency words, high frequency words, and the third predictor consisted of PWs and behavioral errors. The latter predictors were excluded from statistical analyses, respectively. The predictors for the GLM were generated by convolving a Gaussian function with each event (Plichta et al., 2007). To estimate the amplitude of the oxygenation response beta-values for each predictor were calculated by a least squares model fitting procedure maximizing model-to-data fitting (Bullmore et al., 1996). The first and second temporal derivative of each prediction term was included to adapt the onset and dispersion of the model functions to the individual's hemodynamic response. To correct for serial autocorrelated errors resulting from baseline drifts, we fitted a first-order autoregressive process to the error term by the Cochrane-Orcutt procedure (Cochrane and Orcutt, 1949). T-statistics were applied for comparison between response amplitudes. Uncorrected t-values were thresholded at $t(11) \geq 2.2(\alpha=0.05$, two-tailed $)$. We list all channels surviving partial Bonferroni correction in Table 1. For that purpose, the Dubey/Armitage-Parmar alpha boundary was calculated which includes the mean intercorrelation (IC) between the channels (Sankoh et al., 1997). The rationale is that correlated channels must not be treated as independent samples.

Beyond the localization of the oxygenation response we examined the time courses of the changes in [oxy- $\mathrm{Hb}]$ and [deoxy-Hb] by averaging all responses to each condition respectively. The second before stimulus presentation was used as baseline.

To assess the relative hemodynamic response increase to words in comparison to PWs, and to low frequency words in comparison to high frequency words, we simply subtracted the responses of the respectively less active conditions (PWs, high frequency words) from the activating conditions (words, low frequency words). Examples of the resulting time courses are given in Fig. 2.

The rationale to select the examples based on the [deoxy- $\mathrm{Hb}$ ] changes is largely motivated by the physiological link between the changes in [deoxy-Hb] and BOLD contrast. A focal decrease in paramagnetic [deoxy-Hb] is the strongest constituent of a BOLD contrast increase (e.g. Steinbrink et al., 2006). It was thus our intention to link the present work to the fMRI based imaging literature.

To estimate correspondence between channels and cortical topography, Tsuzuki et al.'s (2007) virtual registration method was 

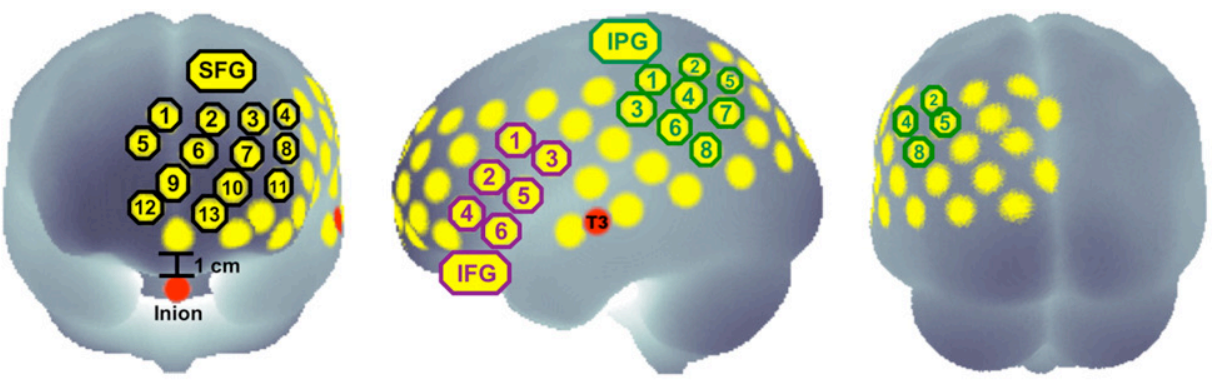

\section{A}
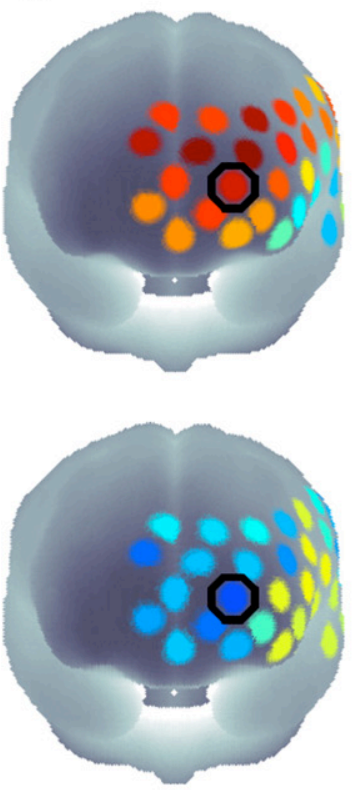

B
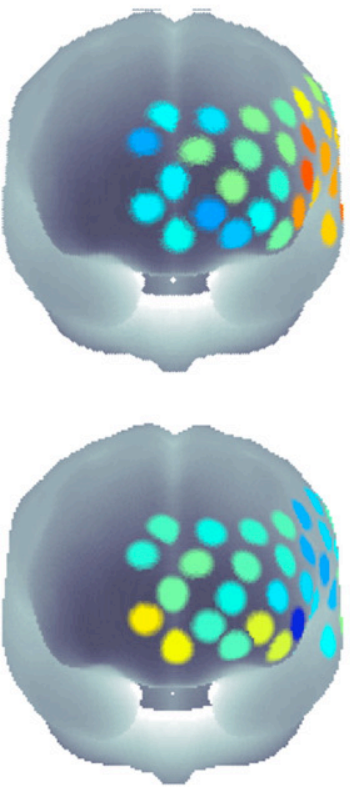

Lexicality (Words > Pseudowords)
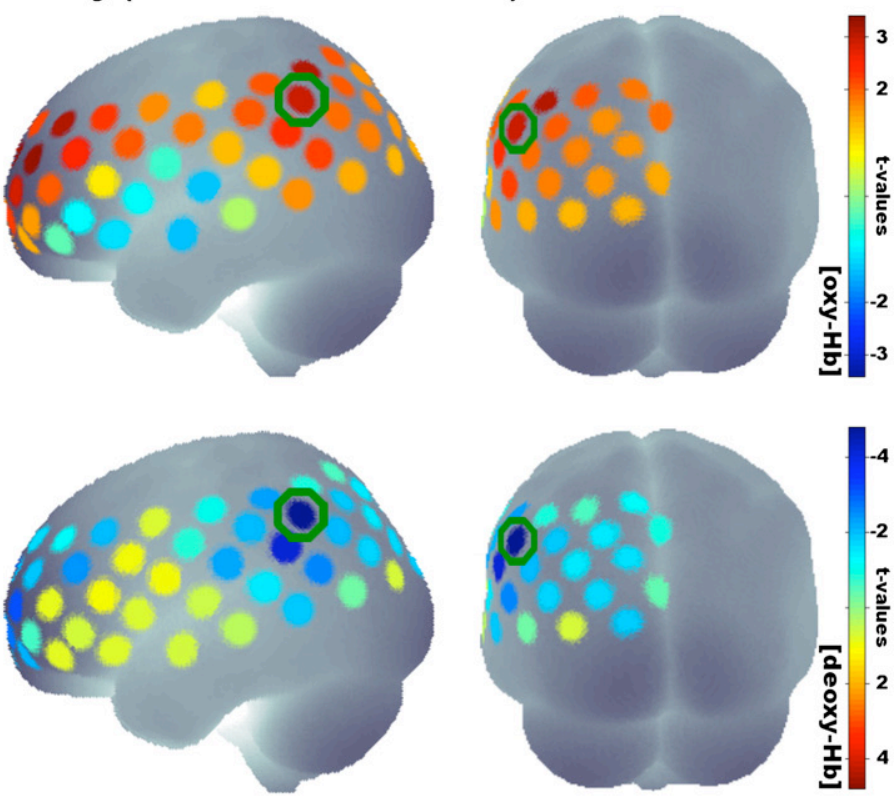

Word Frequency (Low > High)
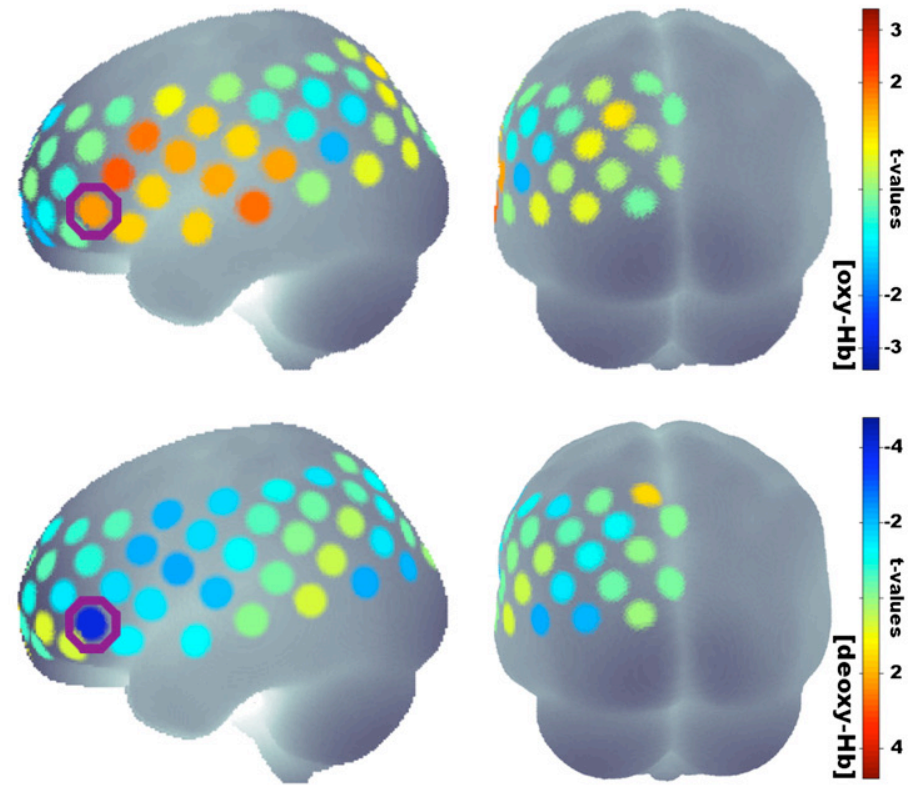

Fig. 1. The upper row indicates the channels belonging to the target regions and the probe set definition. The optode between the middle channels of the lowest optode row was positioned at T3. The lower edge of the probe set was positioned $1 \mathrm{~cm}$ above the inion. Panel A shows the $t$-values for all channels in the lexicality contrast (words $>$ PWs), separately for [oxy-Hb] (upper row) and [deoxy-Hb] (lower row). The time course of channels SFG-10 and IPG-4 are given in Figs. 2A and B, as indicated by the black and green circles, respectively. Panel B shows the t-values for all channels in the word frequency contrast (low $>$ high), separately for [oxy-Hb] (upper row) and [deoxy-Hb] (lower row). The time course of channel IFG-4 is given in Fig. 2C, as indicated by the violet circles. Exact $t$-values of the significant channels can be examined in Table 1. 


\section{A Lexicality (Words $>$ Pseudowords): SFG}

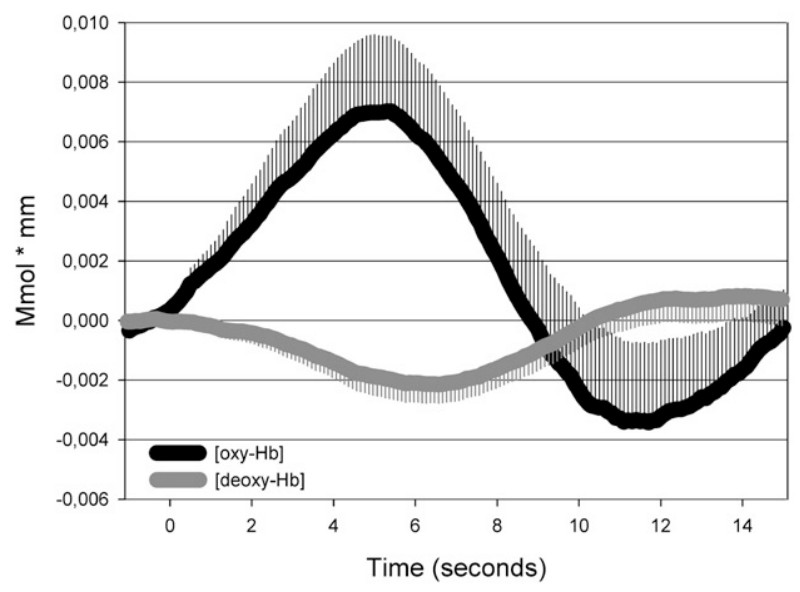

B Lexicality (Words > Pseudowords): IPG

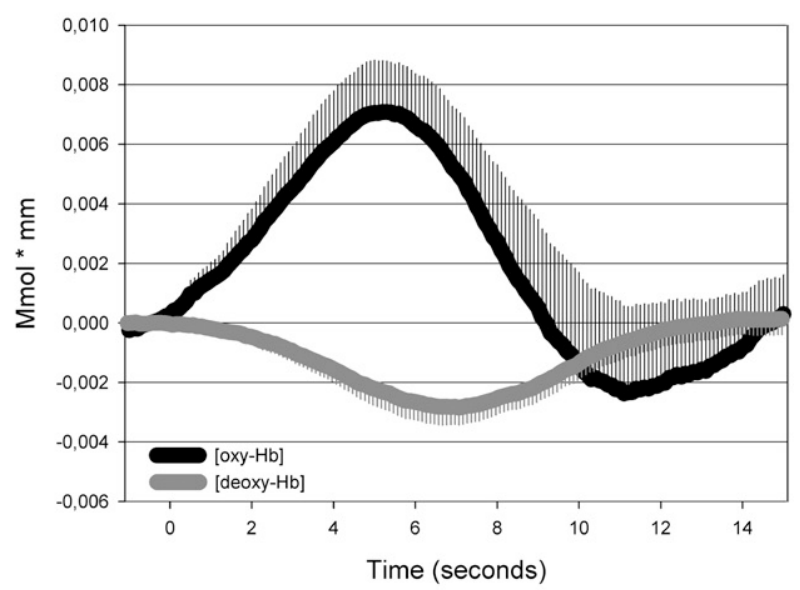

C Word Frequency (Low $>$ High): IFG

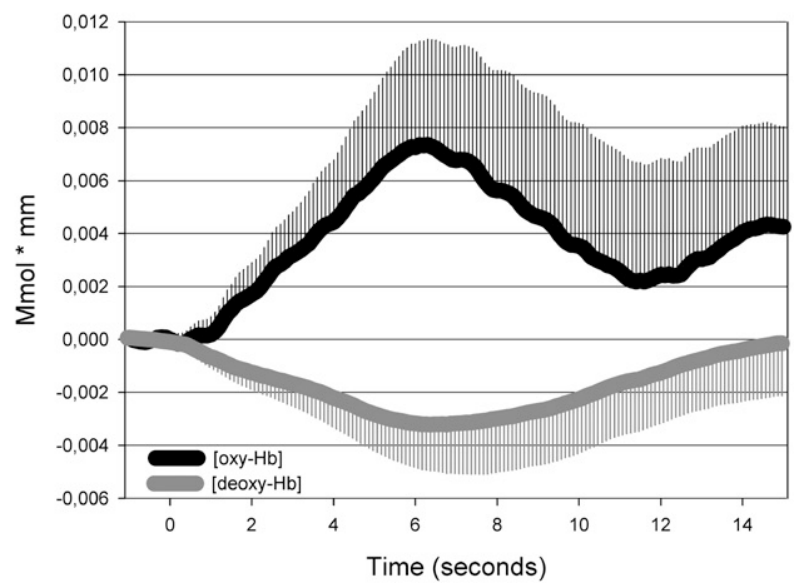

Fig. 2. This figure shows the time courses of the hemodynamic responses exemplified on one channel for each target region. Panels A and B show the time course of the word activation relative to PW activation in channel SFG10 and IPG-4. The time course of the activation of low frequency words relative to high frequency words in channel IFG-4 is given in panel C. Error bars indicate standard errors. applied. This method uses structural information from an anatomical database (Okamoto et al., 2004; Jurcak et al., 2005) to provide estimates of the channel positions in a standardized stereotaxic $3 \mathrm{D}$ brain atlas (Montreal Neurological Institute coordinate system, MNI, Collins et al., 1994; cf. Tsuzuki et al., 2007). It also estimates the spatial uncertainty due to inter-subject variability of the channel locations (freeware available under http://brain.job.affrc.go.jp). The estimated locations were anatomically labeled by means of a Matlab function using anatomical labels from Tzourio-Mazoyer et al.'s (2002) brain atlas. Based on this procedure the following labels for our target regions will be used:

SFG (superior frontal gyrus): left and right medial and middle SFG, and the medial and middle frontal gyrus.

IPG (inferior parietal gyrus): left inferior parietal, angular and the supramarginal gyrus.

IFG (inferior frontal gyrus): includes the left triangular and orbital parts of the IFG.

Channels most probably located in one of those target regions, were indexed from top to bottom and from left to right (cf. Fig. 1, upper row and Table 1).

\section{Results}

\section{Behavioral results}

There was a significant lexicality effect in RTs $(t=5.2 ; p \leq 0.001)$ and error rate $(t=2.8 ; p \leq 0.05)$ with a mean RT of $737 \mathrm{~ms}$ (SD: 257$)$ for words and of $853 \mathrm{~ms}$ (SD: 327) for PW. The mean average error rate was 6.5 (SD: 5.0) for words, and 2.5 (SD: 2.2) for PWs.

A significant effect was also found for word frequency in RTs $(t=4.0, p \leq 0.001)$ and errors $(t=3.2 ; p \leq 0.01)$. Mean RT was $802 \mathrm{~ms}$ (SD: 310) for low frequency words, and $682 \mathrm{~ms}$ (SD: 216) for high frequency words. Corresponding mean error rates were 5.2 (SD: 4.1) for low and 1.3 (SD: 2.0) for high frequency words.

In sum the behavioral results are in line with earlier studies examining behavioral effects of lexicality and word frequency (e.g. Fiebach et al., 2002; Jacobs and Grainger, 1994).

\section{fNIRS (optical topography)}

Changes in [oxy-Hb] and [deoxy-Hb] illustrating the effects of lexicality and word frequency, as well as their anatomical location are given in Fig. 1 and Table 1.

Concerning the effect of lexicality, eleven channels overlying SFG revealed larger [oxy-Hb] increases to words in comparison to PWs (see Table 1 and Fig. 1A). Three of these channels survived the partial Bonferroni correction $(t$ 's $\geq 3.2, \mathrm{IC}=0.55)$. Significant [deoxy-Hb] decreases to words compared to PWs were found in five SFG channels (see Table 1 and Fig. 1A).

Four IPG channels revealed a significant [oxy-Hb] increase to words in comparison to PWs. One of these survived partial Bonferroni correction $(t \geq 3.2, \mathrm{IC}=0.55$, see Fig. $1 \mathrm{~A}$ and Table 1$)$. Five IPG channels revealed a significant [deoxy-Hb] effect. Two of these survived partial Bonferroni correction $(t$ 's $\geq 4.0 ; \mathrm{IC}=0.20)$.

Concerning the effect of word frequency, no significant [oxy-Hb] changes were obtained (maximal $t=2.1, p=0.06$ at channel IFG-2, see Table 1 and Fig. 1B). Significant [deoxy-Hb] decreases were found in two IFG channels. One of these survived partial Bonferroni correction $(t \geq 4.0, \mathrm{IC}=0.18)$. 
Table 1

Channel $t$-values within the regions of interest

\begin{tabular}{|c|c|c|c|c|c|c|}
\hline Channel & & ce & por & & $t$-values & \\
\hline & $X$ & $Y$ & $Z$ & SD & [oxy-Hb] & [deoxy-Hb] \\
\hline
\end{tabular}

Lexicality (Word $>$ Pseudoword)

SFG

\begin{tabular}{rrrrrll}
1 & 6 & 59 & 37 & 11 & $2.4^{*}$ & -1.4 \\
2 & -15 & 59 & 35 & 10 & $2.3^{*}$ & -1.6 \\
3 & -33 & 49 & 35 & 9 & $3.2^{* *}$ & -1.4 \\
4 & -46 & 31 & 38 & 9 & $2,4^{*}$ & -1.9 \\
5 & 14 & 66 & 25 & 11 & $3.1^{*}$ & $-2.8^{*}$ \\
6 & -10 & 67 & 22 & 10 & $3.4^{* *}$ & -1.9 \\
7 & -30 & 61 & 21 & 10 & $3.4^{* *}$ & -1.8 \\
8 & -46 & 44 & 24 & 9 & $2.6^{*}$ & $-2.4^{*}$ \\
9 & 1 & 70 & 10 & 11 & $2.3^{*}$ & -1.9 \\
10 & -23 & 68 & 8 & 9 & $2.9^{*}$ & $-3.1^{*}$ \\
11 & -43 & 55 & 8 & 8 & 2.1 & 0.9 \\
12 & 13 & 71 & -1 & 10 & 1.7 & $-2.2^{*}$ \\
13 & -14 & 70 & -4 & 8 & $2.3^{*}$ & $-2.7^{*}$ \\
IPG & & & & & & \\
1 & -57 & -38 & 53 & 9 & 2.1 & $-2.2^{*}$ \\
2 & -46 & -55 & 58 & 11 & $3.2^{* *}$ & -0.8 \\
3 & -65 & -31 & 41 & 9 & 2.1 & -2.0 \\
4 & -58 & -52 & 47 & 10 & $3.0^{*}$ & $-4.9^{* *}$ \\
\hline 5 & -42 & -69 & 51 & 11 & 2.1 & -1.8 \\
6 & -65 & -46 & 34 & 9 & $2.4^{*}$ & $-4.1^{* *}$ \\
7 & -53 & -67 & 40 & 10 & 2.0 & -2.0 \\
8 & -62 & -60 & 24 & 9 & $2.3^{*}$ & $-2.5^{*}$
\end{tabular}

Word Frequency (Low $>$ High)

IFG

\begin{tabular}{rrrrrrl}
1 & -56 & 21 & 28 & 9 & 1.9 & -2.0 \\
2 & -55 & 33 & 12 & 8 & 2.1 & -1.5 \\
3 & -63 & 6 & 20 & 8 & 1.5 & $-2.2^{*}$ \\
4 & -52 & 44 & -3 & 8 & 1.6 & $-4.0^{* *}$ \\
5 & -59 & 18 & 5 & 9 & 1.2 & -1.5 \\
6 & -53 & 29 & 11 & 11 & 1.2 & -1.3 \\
\hline
\end{tabular}

Channel numbers, MNI coordinates, estimated inter-subject variability (SD) and significant uncorrected t-values ( $d f=11, p$ 's $\leq 0.05$, two-tailed) are given. Significant channels are marked by $*\left(t^{\prime} \mathrm{s} \geq 2.2\right)$. $t$-values surviving partial Bonferroni correction are marked by ** ( $t^{\prime} \mathrm{s} \geq 3.2$ for [oxy-Hb] and $t^{\prime} \mathrm{s} \geq 4.0$ for $[$ deoxy-Hb] due to channel intercorrelations). For shaded channels exemplary time courses are given in Fig. 2.

Beyond the statistical comparison between conditions we also examined the time course of the oxygenation responses. Fig. 2 provides examples for the cortical target regions. For the selection of the example channels we chose those channels in the target regions, which showed the most significant decrease in [deoxy- $\mathrm{Hb}]$. The rationale to select these channels based on [deoxy-Hb] changes is twofold: (i) the signal of the most widely used functional imaging technique, BOLDcontrast fMRI, relies on changes in focal susceptibility elicited by decreases in [deoxy-Hb] (Ogawa et al., 1990), thus we consider [deoxy-Hb] changes the best parameter to link our data to the existing imaging literature (Kleinschmidt et al., 1996; Steinbrink et al., 2006). (ii) The fine-tuned regulation of blood flow velocity and blood volume changes (e.g. Buxton et al., 1998) is specific to the cerebral vasculature. This may explain, why [oxy-Hb] changes are more sensitive to extracerebral contamination as has been demonstrated in an experiment using a simple motor paradigm (Boden et al., 2007).

Fig. 2A provides the time course of the hemodynamic response of channel SFG-10 anatomically corresponding to the left SFG $(x / y / z$ : $-23 / 68 / 8$ in MNI-space). The differential response (i.e. response to words minus response to $\mathrm{PW}$ ) demonstrates that the response pattern is in line with previous publications showing a decrease in [deoxy-Hb] accompanied by a larger increase in [oxy-Hb]. A similar difference was seen over the IPG for this comparison (words vs. PWs). Fig. 2B illustrates the differential time course in this region (IPG-4; $x / y / \mathrm{z}$ : $-47 /-52 / 47$ in MNI-space). Finally, an example for the comparison between low versus high frequency words is given in Fig. 2C. Here the response to high frequency words was subtracted from that to low frequency words. Again a stronger increase in [oxy-Hb] and a more pronounced decrease in [deoxy-Hb] is clearly seen to low frequency words in this region corresponding to left IFG (IFG-4; $x / y / z: 35 /-52 /-3$ in MNI-space). The response pattern seen over all three channels is explicable by an increase in blood volume and a faster washout of [deoxy-Hb], the latter corresponding to an increase in BOLD-contrast (Kleinschmidt et al., 1996). Hence our findings show greater activations for words (vs. PW) over the left SFG and IPG, while a larger activation over the left IFG is found for low frequency when compared to high frequency words.

\section{Discussion}

The present study shows that neuronal correlates of visual word recognition can be investigated by optical topography. Specifically it was demonstrated that both, lexicality and word-frequency yield differential patterns of cortical activation. The major findings are: (i) words elicit a statistically larger activation in the SFG and IPG when compared to PWs (lexicality effect). This is indicated by an increase in [oxy-Hb] and a decrease in [deoxy-Hb]. (ii) Low frequency words elicit a greater activation in the IFG when compared to high frequency words (word frequency effect), as indicated by a decrease of [deoxy-Hb]. Moreover, we demonstrated that more than one target region can be assessed by fNIRS within the same effect, and that the functional specificity of neighboring regions can be assessed by applying the probabilistic mapping method.

These findings supply functional neuroimaging support for two models, the Multiple Read Out Model (MROM, Grainger and Jacobs, 1996) and the dual route model (DRM, Coltheart et al., 2001), both of which were originally proposed for explaining behavioral data. While our findings are generally in line with previous imaging studies, the discussion will also focus on the novel methodological approach.

\section{The Lexicality effect}

We show a statistically larger [oxy-Hb] increase and [deoxy-Hb] decrease to words in comparison to PWs for the channels overlying the SFG. This activation difference of the SFG during visual word recognition is predicted by the MROM (Grainger and Jacobs, 1996; Jacobs et al., 1998; Fiebach et al., 2007) which posits that different decision mechanisms for words and PWs will be activated: A "yes" response is generated by intra-lexical activation criteria while the "no" response is generated by an extralexical temporal threshold mechanism (cf. Fiebach et al., 2007). Thus the greater RT for PWs can be explained by the temporal threshold, and the 'no-response' relies on a lesser SFG activation. Previous neuroimaging studies reported similar findings. In a PET study Price (2000) demonstrated an increase in blood volume in the SFG and these findings were confirmed by successive fMRI studies reporting on BOLD-contrast increases in the SFG (Binder et al., 2003; Ischebeck et al., 2004; Kuchinke et al., 2005; for a review of the role of the SFG in decision-related processes also see Ridderinkhof et al., 2004). Finally the SFG's role in generating a lexical decision is supported by the finding that word/ 
pseudoword differentiation during silent articulation without overt judgment on lexicality does not activate SFG (cf. e.g. Cohen et al., 2003; Kronbichler et al., 2004).

An alternative explanation of the SFG effect observed in the present and the previous imaging studies refers to differences in semantic retrieval. According to this hypothesis the increased SFG activation to words results from control functions with regard to retrieval of semantic information (cf. Binder et al., 1999, 2003).

Principally our findings might indicate that task difficulty accounts for the SFG effect. Words not only elicited a greater SFG activation than PWs, but also more errors. Error processing has been assumed to rely on the anterior cingulate (Yeung et al., 2004), but also on the mediofrontal gyrus (Ridderinkhof et al., 2004), which is part of the SFG. Though actual errors were excluded from the analyses, Yeung et al. propose that errors may be activated partially, even when a correct response is generated. Thus items more likely to elicit an error may induce the SFG activation due to partial error activation. To test whether partial error processing is the relevant influence for the decision-related activation in the SFG, we added a fourth predictor in our GLMs representing the amount of errors per item. If partial error activation was the relevant factor for the differential SFG activation we would expect to eliminate the effect seen in our analysis reported above. On the contrary our results did not change qualitatively. Still statistically significant differences were obtained in the SFG (cf. Appendix A). Thus we conclude that the larger SFG activation found in response to words in comparison to $\mathrm{PWs}^{2}$ is at least partially independent from error processing. This challenges models of visual word recognition based on behavioral measures alone (e.g. Grainger and Jacobs, 1996; but see e.g. Braun et al., 2006; Hofmann et al., 2008).

In the channels overlying the IPG words also elicited a stronger hyperoxygenation when compared to PWs (Figs. 1A and 2B; Table 1). The role of IPG in lexical decision has been previously reported. A PET study reports blood volume increases to words in comparison to PWs (Price, 2000) while greater BOLD responses in IPG were obtained in response to similar paradigms, though this finding is controversial (Binder et al., 2003; Cohen et al., 2003; Ischebeck et al., 2004; Kuchinke et al., 2005; but see Fiebach et al., 2002).

Conceptually Dejerine (1891) was the first to claim this region's role for 'memories' of the visual word form. Nowadays this concept has been further elaborated in the framework of different theories. Apart from whole word form representations, it was proposed that sublexical representations exist even at the level of the syllable (Goswami and Ziegler, 2006). When words are tested against consonant strings (Cohen et al., 2003), an alternate explanation for the IPG effect of lexicality can be discussed. In that case syllabic representation rather than semantic load may explain the difference in IPG activation (cf. Owen et al., 2004). Even though we cannot fully exclude the possibility of syllabic representation to drive IPG, we suggest - in line with Binder et al. $(1999,2003)$ - that the critical difference between words and PWs is the semantic representation exclusive to words, a difference which dominates the differential IPG activation for lexicality. This conclusion receives support from lesion

\footnotetext{
${ }^{2}$ The other main effects also remained unchanged. A larger change in [oxy$\mathrm{Hb}$ ] and [deoxy-Hb] over the IPG was observed to words in comparison to PWs. Low frequency words elicited a larger [deoxy-Hb] response than high frequency words. To control for task difficulty, we finally used the RT of each trial to control for activations purely due to varying unspecific task effects. Again, the main results described in our original analysis remained unchanged and statistically significant (cf. Appendix A).
}

studies of the IPG (see Price, 2000 for an overview), and is in line with the concept proposed by BOLD-contrast findings in studies on dyslexia (Booth et al., 2004; Horwitz et al., 1998; Pugh et al., 2000).

In sum our study provides evidence that the IPG plays a key role in the integration not only of orthographic and phonological information, but actively links these to semantic information.

\section{The word frequency effect}

While [oxy-Hb] changes only showed a trend, the word frequency effect elicited significant differences between low and high frequency words for the decrease in [deoxy-Hb] (see Table 1 and Fig. 1B). Two channels overlying the IFG showed a larger decrease in [deoxy-Hb] in response to the low frequency words. One of these survived the Bonferroni correction. Note that a decrease in [deoxy-Hb] corresponds to an increase in BOLD-contrast (Kleinschmidt et al., 1996). Hence, the larger decrease in [deoxy-Hb] can be interpreted as an indicator of a stronger underlying neuronal response in analogy to studies relying on BOLD-contrast fMRI (see Fig. 2C, and below for the discussion of the NIRS response).

The $[$ deoxy-Hb] finding confirms the most established BOLD effect in fMRI research on word recognition (Carreiras et al., 2006; Fiebach et al., 2002, 2003; Ischebeck et al., 2004; Kronbichler et al., 2004; Nakic et al., 2006; Prabhakaran et al., 2006). The established interpretation is that grapheme-phoneme correspondences are being computed in the IFG. An alternative account would propose that identification of low frequency words is more equivocal than that of high frequency words. Therefore several semantic candidates are activated, and the left IFG's role is to select between these preactivated candidates (Thompson-Schill et al., 1997).

Although our results are in line with previous fMRI studies, it should be noted that the present stimulus material was even more rigidly controlled for potentially confounding effects. In order to quantify familiarity with the letter strings in terms of an aggregation of more or less familiar features, Kronbichler et al. (2004) controlled for bigram frequency. The present study used type and token bigram frequencies. Type indicates the amount of words in which a specific bigram occurs, while token denotes the summed frequencies of these words (cf. Hofmann et al., 2007). Type measures can be considered to quantify the familiarity of all existing words assuming they are equally activated. Token measures, in contrast, consider the actual likelihood to be activated, because they reflect word frequencies. Therefore, the alternative explanation of a global familiarity with the words as an aggregation of more or less familiar features can be ruled out based on the present study.

\section{Optical topography as a tool to investigate visual word recognition}

Owing to its low constraints on the experimental environment fNIRS, a silent method, is an exquisite tool to investigate language and higher cognition, in which an MRI environment may induce substantial distortion of behavior and speech perception. Like EEG it may also more easily find applications in psychiatric (Fallgatter et al., 2004) and neuropsychological patients (Zabel and Chute, 2002) and has already been established as a tool in studying neuropsychological development in neonates and infanthood (Pena et al., 2003; Taga et al., 2003; Wartenburger et al., 2007). However, the appraisal of these advantages must face the fact that NIRS cannot supply the exquisite and ever increasing spatial resolution of fMRI-based approaches. Like EEG any anatomical inference on the cortical areas relies on external bony landmarks and can thus be 
referenced to the 10-20 system and its extensions. Recently, a series of publications has addressed this issue and supplied a tool to frame the very rough anatomical differentiation into a probabilistic mapping (Jurcak et al., 2005, 2007; Okamoto et al., 2004). In brief, cortical anatomy was related to landmarks identified by 10-20system positions (Tsuzuki et al., 2007) in 1000 simulated brains. The tool therefore supplies a measure of the likelihood of a specific position on the subject's skull to correspond to a specific cortical area and can supply the corresponding MNI-space coordinates. We are fully aware of the limitations of such a reference system and its potential source of error, hence without doubt NIRS will always have to respect functional anatomical facts assessed with methods of superior spatial resolution. Nonetheless the present findings demonstrate that the localization procedure yields the very cortical regions previously discussed by functional imaging studies of word recognition. By contrasting the lexicality and the word frequency effect it is apparent that spatially distinct areas are activated (see Fig. 1). This we consider a sound basis to address developmental aspects of lexical processing, e.g. in children learning to read.

There is yet another issue which is somewhat controversial concerning the interpretation of fNIRS signals. Accepted models of neuro-vascular coupling posit that the increase in $\mathrm{rCBF}$ is disproportional to the increase in oxygen consumption when a cortical area is activated (Buxton et al., 2004; Fox and Raichle, 1986; Huppert et al., 2007). The resulting hyperoxygenation can be measured by NIRS, assessing changes in [oxy-Hb] and [deoxy-Hb]. It is, however, controversial whether an $\mathrm{rCBF}$ increase could potentially rely selectively on an increase in blood volume due to arterial dilation without an accelerated flow velocity. Moreover a number of dynamic flow-volume relationships were discussed to explain non-linearities of the vascular response governing the BOLD contrast (Mandeville et al., 1999). In case of a pure volume increase [deoxy-Hb] might either show no change or even a slight increase due to its production by oxidative metabolism and the fact that even arterial blood contains a quantity of deoxygenated hemoglobin. This may account for the observation, that more $[\mathrm{oxy}-\mathrm{Hb}]$ channels revealed significant changes than $[\mathrm{deoxy}-\mathrm{Hb}]$ channels. The channels showing [oxy-Hb] changes but no [deoxy-Hb] changes may be an activation BOLD-contrast fMRI would be 'blind' to, because the decrease in paramagnetic [deoxy-Hb] is the primary source of BOLD-contrast increases (Huppert et al., 2007; Kleinschmidt et al., 1996; Ogawa et al., 1990).

Such observations have regularly led to the additional appraisal as to the superiority of NIRS, assessing oxygenation and volume changes, the latter by summing the changes in both compounds. In the present study we find increases in [oxy-Hb] and decreases in [deoxy$\mathrm{Hb}$ ] for the lexicality effect in the SFG and IPG. In contrast, word frequency did not elicit a statistically significant [oxy-Hb] increase in any of the channels. Interestingly an early PET study relying on blood volume changes yielded also only a tendency towards greater blood volume changes for low frequency words (Fiez et al., 1999), whereas successive fMRI studies reliably and reproducibly demonstrated the difference for BOLD-contrast changes. This might be interpreted as an indicator of vascular differences between different cortical areas as has been discussed previously (Blood et al., 2002). However, we favor the alternative explanation that [oxy-Hb] and [deoxy-Hb] changes in NIRS are subject to different signal to noise ratios. The fact that extracerebral, i.e. systemic, hemoglobin changes particularly affect [oxy- $\mathrm{Hb}]$ has been stressed recently (Boden et al., 2007). At least for motor tasks increases in heart rate were reported to coincide with the stimulation period (Franceschini et al., 2003). In the present study further evidence for the influence of the systemic response specifically on $[\mathrm{oxy}-\mathrm{Hb}]$ can be derived from the observation that for [oxy-Hb] intercorrelations between channels amount to more than twice of that observed for [deoxy-Hb]. Moreover, the largest $\mathrm{t}$-values were obtained for [deoxy-Hb]. This may indicate its better signal-to-noise ratio.

In sum, the changes in oxygenation are well in line with the model of neurovascular coupling that is the basis of all vascular based methodologies, most prominently BOLD contrast fMRI. Though localization of fNIRS is limited, we demonstrate that specific sub-processes can be reliably differentiated by the topographical approach and can be tentatively framed in a common reference system to be compared to fMRI or PET studies.

Future studies will have to critically evaluate the versatility of such an approach in the development of literacy in children and its impairment in neuropsychiatric syndromes. Results from work on language perception in adults and language development in infanthood based on spoken language are extremely encouraging with respect to apply the method for this endeavor. It should be noted also that the present study is the first in language research using fNIRS to identify three distinct areas within one hemisphere and to reliably differentiate their respective roles during lexical decision.

\section{Acknowledgments}

We would like to thank Theresa Huter for assistance during data collection, the participants that partook voluntarily, and the Hitachi Medical Corporation for providing the ETG-4000 equipment. This work was partly supported by grants by the Deutsche Forschungsgemeinschaft (research unit "Zwischen Interferenz und Optimierung: Konflikte als Signale in kognitiven Systemen”, TP 3 Jacobs, JA 823/ 4-1; "Zur Rolle phonologischer Prozesse beim Lesen komplexer Woerter. Ein sprachvergleichender Ansatz", JA 823/3-1/Jacobs; and clinical research unit "Aufmerksamkeitsdefizit-/Hyperaktivitaetssyndrom (ADHS): Molekulare Pathogenese und Endophaenotypen im Therapieverlauf", TP 8, Fallgatter, KFO-125/1), the Berlin NeuroImaging Center, the Bernstein Center for Computational Neuroscience Berlin, the EU (NEST 012778, EFRE 20002006 2/6), the Grant-inAid for Scientific Research (18390404, 19650079, I. Dan), and the Program for Promotion of Basic Research Activities for Innovative Bioscience (PROBRAIN, I. Dan).

\section{Appendix A}

Channel t-values for regions of interest when errors or RTs were held constant

\begin{tabular}{|c|c|c|c|c|}
\hline \multirow[t]{2}{*}{ Channel } & \multicolumn{2}{|c|}{$T$-values (errors constant) } & \multicolumn{2}{|c|}{$T$-values (RTs constant) } \\
\hline & [oxy-Hb] & [deoxy-Hb] & [oxy-Hb] & {$[$ deoxy-Hb] } \\
\hline \multicolumn{5}{|c|}{ Lexicality (Word $>$ Pseudoword) } \\
\hline \multicolumn{5}{|l|}{ SFG } \\
\hline 1 & $2.2^{*}$ & -1.7 & 1.7 & -1.6 \\
\hline 2 & 1.5 & -1.2 & 1.3 & -1.1 \\
\hline 3 & $2.7^{*}$ & $-2.2 *$ & $2.4^{*}$ & -2.0 \\
\hline 4 & $3.5^{* *}$ & -1.3 & $3.3^{* *}$ & -1.2 \\
\hline 5 & $2.9 *$ & $-2.4^{*}$ & $2.3^{*}$ & -2.1 \\
\hline 6 & $3.2^{* *}$ & $-3.5^{*}$ & $2.7^{*}$ & $-3.7^{*}$ \\
\hline 7 & $3.0^{*}$ & -1.9 & $2.7^{*}$ & -1.7 \\
\hline 8 & $2.9^{*}$ & -1.7 & $2.3^{*}$ & -1.7 \\
\hline 9 & $2.6^{*}$ & $-3.3 *$ & $2.4 *$ & $-3.1 *$ \\
\hline 10 & $3.6^{* *}$ & $-2.7^{*}$ & $3.2 * *$ & $-2.6^{*}$ \\
\hline 11 & $2.3^{*}$ & 0.4 & 1.9 & 0.9 \\
\hline 12 & $2.8^{*}$ & -1.7 & 2.0 & -1.9 \\
\hline 13 & $3.8^{* *}$ & $-3.2 *$ & $3.4^{* *}$ & $-3.4^{*}$ \\
\hline
\end{tabular}

(continued on next page) 


\begin{tabular}{|c|c|c|c|c|}
\hline \multirow[t]{2}{*}{ Channel } & \multicolumn{2}{|c|}{$T$-values (errors constant) } & \multicolumn{2}{|c|}{$T$-values (RTs constant) } \\
\hline & [oxy-Hb] & {$[$ deoxy-Hb] } & {$[\mathrm{oxy}-\mathrm{Hb}]$} & {$[$ deoxy-Hb] } \\
\hline \multicolumn{5}{|c|}{ Lexicality (Word > Pseudoword) } \\
\hline \multicolumn{5}{|c|}{ ( } \\
\hline 1 & 2.1 & -1.8 & 1.9 & -1.9 \\
\hline 2 & $2.7^{*}$ & $-3.3^{*}$ & $2.5^{*}$ & $-3.1^{*}$ \\
\hline 3 & 2.1 & -1.7 & 1.9 & -1.7 \\
\hline 4 & $2.9^{*}$ & $-3.6^{*}$ & $2.8^{*}$ & $-3.4^{*}$ \\
\hline 5 & $3.9^{* *}$ & $-2.8^{*}$ & $3.2^{* *}$ & $-2.8^{*}$ \\
\hline 6 & $2.4^{*}$ & $-3.0^{*}$ & 2.0 & $-2.9^{*}$ \\
\hline 7 & $3.9^{* *}$ & $-2.7^{*}$ & $3.4^{* *}$ & $-2.7^{*}$ \\
\hline 8 & $3.1^{*}$ & $-2.2^{*}$ & $2.7 *$ & -1.8 \\
\hline \multicolumn{5}{|c|}{ Word Frequency (Low $>$ High) } \\
\hline \multicolumn{5}{|c|}{ IFG } \\
\hline 1 & 1.4 & -1.6 & 1.4 & -1.6 \\
\hline 2 & 1.6 & -1.5 & 1.5 & -1.5 \\
\hline 3 & 1.0 & -2.0 & 1.0 & $-2.2^{*}$ \\
\hline 4 & 0.9 & $-3.0^{*}$ & 0.8 & $-2.9^{*}$ \\
\hline 5 & 0.6 & -1.4 & 0.6 & -1.4 \\
\hline 6 & 0.6 & -1.1 & 0.6 & -1.0 \\
\hline
\end{tabular}

Channel numbers and significant uncorrected t-values $(d f=11 . p \mathrm{~s} \leq 0.05$. two-tailed) in the target region channels are given (cf. Table 1).

\section{References}

Baayen, R.H., Piepenbrock, R., Gulikers, L., 1995. The CELEX lexical database (CD-ROM). Linguistic Data Consortium, University of Pennsylvania, Philadelphia.

Binder, J.R., Frost, J.A., Hammeke, T.A., Bellgowan, P.S.F., Rao, S.M., Cox, R.W., 1999. Conceptual processing during the conscious resting state: A functional fMRI study. J. Cogn. Neurosci. 11, 80-95.

Binder, J.R., McKiernan, K.A., Parsons, M.E., Westbury, C.F., Possing, E.T., Kaufman, J.N., Buchanan, L., 2003. Neural correlates of lexical access during visual word recognition. J. Cogn. Neurosci. 15, 372-393.

Blood, A.J., Pouratian, N., Toga, A.W., 2002. Temporally staggered forelimb stimulation modulates barrel cortex optical intrinsic signal responses to whisker stimulation. J. Neurophysiol. 88, 422-437.

Boden, S., Obrig, H., Koehncke, C., Benav, H., Koch, S.P., Steinbrink, J., 2007. The oxygenation response to functional stimulation: is there a physiological meaning to the lag between parameters? NeuroImage 36, 100-107.

Booth, J.R., Burman, D.D., Meyer, J.R., Gitelman, D.R., Parrish, T.B., Marsel Mesulam, M., 2004. Development of Brain Mechanisms for Processing Orthographic and Phonologic Representations. J. Cogn. Neurosci. 16, 1234-1249.

Braun, M., Jacobs, A., Hahne, A., Ricker, B., Hofmann, M., Hutzler, F., 2006. Model-generated lexical activity predicts graded ERP amplitudes in lexical decision. Brain Res. 1073-1074, 431-339.

Brunswick, N., McCrory, E., Price, C.J., Frith, C.D., Frith, U., 1999. Explicitly and implicit processing of words and pseudowords by adult developmental dyslexics. Brain 122, 1901-1917.

Bullmore, E., Brammer, M., Williams, S.C., Rabe-Hesketh, S., Janot, N., David, A., Mellers, J., Howard, R., Sham, P., 1996. Statistical methods of estimation and inference for functional MR image analysis. Magn. Reson. Med. 35, 261-277.

Buxton, R., Wong, E., Frank, L., 1998. Dynamics of blood flow and oxygenation changes during brain activation: the balloon model. Magn. Reson. Med. 39, 855-864.

Buxton, R.B., Uludag, K.M., Dubowitz, D.J., Liu, T.T., 2004. Modeling the hemodynamic response to brain activation. NeuroImage 23 (Suppl 1), S220-S233.

Carreiras, M., Mechelli, A., Price, C.J., 2006. Effect of word and syllable frequency on activation during lexical decision and reading aloud. Hum. Brain Mapp. 27, 963-972.
Cochrane, D., Orcutt, G., 1949. Application of least squares regression to relationships containing autocorrelated error terms. J. Am. Stat. Assoc. $44,32-61$.

Cohen, L., Martinaud, O., Lemer, C., Lehericy, S., Samson, Y., Obadia, M., Slachevsky, A., Dehaene, S., 2003. Visual Word Recognition in the Left and Right Hemispheres: Anatomical and Functional Correlates of Peripheral Alexias. Cereb. Cortex 13, 1313-1333.

Collins, D.L., Neelin, P., Peters, T.M., Evans, A.C., 1994. Automatic 3D intersubject registration of MR volumetric data in standardized Talairach space. J. Comput. Assist. Tomogr. 18, 192-205.

Coltheart, M., Rastle, K., Perry, C., Langdon, R., Ziegler, J., 2001. DRC: A dual route cascaded model of visual word recognition and reading aloud. Psychol. Rev. 108, 204-256.

Cope, M., Delpy, D.T., 1988. System for long-term measurement of cerebral blood and tissue oxygenation on newborn infants by near infra-red transillumination. Med. Biol. Eng. Comput. 26, 289-294.

Damasio, A.R., Damasio, H., 1983. The anatomic basis of pure alexia. Neurology 33, 1573-1583.

Dejerine, J., 1891. Sur un cas de cecite verbal avec agraphie suivi d'autopsie. Mem. Soc. Biol. 3, 197-201.

Fallgatter, A.J., Mueller, T.J., Strik, W.K., 1998. Prefrontal hypooxygenation during language processing assessed with near-infrared spectroscopy. Neuropsychobiology 37, 215-218.

Fallgatter, A.J., Ehlis, A.C., Wagener, A., Michel, T., Herrmann, M.J., 2004 Near-infrared spectroscopy in psychiatry. Nah-Infrarot Spektroskopie in der Psychiatrie. Nervenarzt 75, 911-916.

Fiebach, C.J., Friederici, A.D., Mueller, K., Von Cramon, D.Y., 2002. fMRI evidence for dual routes to the mental lexicon in visual word recognition. J. Cogn. Neurosci. 14, 11-23.

Fiebach, C.J., Friederici, A.D., Mueller, K., Von Cramon, D.Y., Hernandez, A.E., 2003. Distinct brain representations for early and late learned words. NeuroImage 19, 1627-1637.

Fiebach, C.J., Ricker, B., Friederici, A.D., Jacobs, A.M., 2007. Inhibition and facilitation in visual word recognition: Prefrontal contribution to the orthographic neighborhood size effect. NeuroImage 36, 901-911.

Fiez, J.A., Balota, D.A., Raichle, M.E., Petersen, S.E., 1999. Effects of lexicality, frequency, and spelling-to-sound consistency on the functional anatomy of reading. Neuron 24, 205-218.

Fox, P.T., Raichle, M.E., 1986. Focal physiological uncoupling of cerebral blood flow and oxidative metabolism during somatosensory stimulation in human subjects. Proc. Natl. Acad. Sci. U. S. A. 83, $1140-1144$.

Franceschini, M.A., Fantini, S., Thompson, J.H., Culver, J.P., Boas, D.A., 2003. Hemodynamic evoked response of the sensorimotor cortex measured noninvasively with near-infrared optical imaging. Psychophysiology 40, $548-560$.

Geschwind, N., 1965. Disconnexion syndromes in animals and man. Brain $88,237-294$

Goswami, U., Ziegler, J.C., 2006. A developmental perspective on the neural code for written words. Trends Cogn. Sci. 10, 142-143.

Grainger, J., Jacobs, A.M., 1996. Orthographic processing in visual word recognition: A multiple read-out model. Psychol. Rev. 103, 518-565.

Hart, J., Gordon, B., 1990. Delineation of single-word semantic comprehension deficits in aphasia, with anatomical correlation. Ann. Neurol. 27, 226-231.

Herrmann, M.J., Walter, A., Ehlis, A.-C., Fallgatter, A.J., 2006. Cerebral oxygenation changes in the prefrontal cortex: Effects of age and gender. Neurobiol. Aging 27, 888-894.

Hofmann, M.J., Stenneken, P., Conrad, M., Jacobs, A.M., 2007. Sublexical frequency measures for orthographic and phonological units in German. Behav. Res. Meth. Instrum. 39, 620-629.

Hofmann, M.J., Tamm, S., Braun, M.M., Dambacher, M., Hahne, A., jacobs, AM., 2008. Conflict monitoring engages the mediofrontal cortex during nonword processing. NeuroReport 19, 25-29.

Homae, F., Watanabe, H., Nakano, T., Asakawa, K., Taga, G., 2006. The right hemisphere of sleeping infant perceives sentential prosody. Neurosci. Res. 54, 276-280. 
Horovitz, S.G., Gore, J.C., 2004. Simultaneous event-related potential and near-infrared spectroscopic studies of semantic processing. Hum. Brain Mapp. 22, 110-115.

Horwitz, B., Rumsey, J.M., Donohue, B.C., 1998. Functional connectivity of the angular gyrus in normal reading and dyslexia. Proc. Natl. Acad. Sci. 95, 8939-8944

Huppert, T.J., Allen, M.S., Benav, H., Jones, P.B., Boas, D.A., 2007. A multicompartment vascular model for inferring baseline and functional changes in cerebral oxygen metabolism and arterial dilation. J. Cereb. Blood Flow Metab. 27, 1262-1279.

Ischebeck, A., Indefrey, P., Usui, N., Nose, I., Hellwig, F., Taira, M., 2004 Reading in a regular orthography: An fMRI study investigating the role of visual familiarity. J. Cogn. Neurosci. 16, 727-741.

Jacobs, A.M., Graf, R., Kinder, A., 2003. Receiver operating characteristics in the lexical decision task: Evidence for a simple signal-detection process simulated by the multiple read-out model. J. Exper. Psychol., Learn., Mem., Cogn. 29, 481-488.

Jacobs, A.M., Grainger, J., 1994. Models of visual word recognition: Sampling the state of the art. J. Exp. Psychol. Hum. Percept. Perform. 20, 1311-1334

Jacobs, A.M., Rey, A., Ziegler, J.C., Grainger, J., 1998. MROM-p: An interactive activation, multiple readout model of orthographic and phonological processes in visual word recognition. In: Grainger, J., Jacobs, A.M. (Eds.), Localist connectionist approaches to human cognition. Lawrence Erlbaum Associates Inc., pp. 147-188.

Jasper, H.H., 1958. Report to the committee on methods and clinical examination in electroencephalography. Electroencephalogr. Clin. Neurophysiol. $10,371-375$.

Jurcak, V., Okamoto, M., Singh, A., Dan, I., 2005. Virtual 10-20 measurement on MR images for inter-modal linking of transcranial and tomographic neuroimaging methods. NeuroImage 26, 1184-1192.

Jurcak, V., Tsuzuki, D., Dan, I., 2007. 10/20, 10/10, and 10/5 systems revisited: Their validity as relative head-surface-based positioning systems. NeuroImage 34, 1600-1611.

Kleinschmidt, A., Obrig, H., Requardt, M., Merboldt, K.D., Dirnagl, U., Villringer, A., Frahm, J., 1996. Simultaneous recording of cerebral blood oxygenation changes during human brain activation by magnetic resonance imaging and near-infrared spectroscopy. J. Cereb. Blood Flow Metab. 16, 817-826.

Kronbichler, M., Hutzler, F., Wimmer, H., Mair, A., Staffen, W., Ladurner, G., 2004. The visual word form area and the frequency with which words are encountered: Evidence from a parametric fMRI study. NeuroImage 21, 946-953.

Kronbichler, M., Hutzler, F., Staffen, W., Mair, A., Ladurner, G., Wimmer, H., 2006. Evidence for a dysfunction of left posterior reading areas in German dyslexic readers. Neuropsychologia 44, 1822-1832.

Kuchinke, L., Jacobs, A.M., Grubich, C., Vo, M.L.-H., Conrad, M., Herrmann, M., 2005. Incidental effects of emotional valence in single word processing: An fMRI study. NeuroImage 28, 1022-1032.

Mandeville, J.B., Marota, J.J., Ayata, C., Zaharchuk, G., Moskowitz, M.A., Rosen, B.R., Weisskoff, R.M., 1999. Evidence of a cerebrovascular pos arteriole wind kessel with delayed compliance. J. Cereb. Blood Flow Metab. 19, 679-689.

Nakic, M., Smith, B.W., Busis, S., Vythilingam, M., Blair, R.J.R., 2006. The impact of affect and frequency on lexical decision: The role of the amygdala and inferior frontal cortex. NeuroImage 31, 1752-1761.

Noguchi, Y., Takeuchi, T., Sakai, K.L., 2002. Lateralized activation in the inferior frontal cortex during syntactic processing: Event-related optical topography study. Hum. Brain Mapp. 17, 89-99.

Obrig, H., Villringer, A., 2003. Beyond the visible-imaging the human brain with light. J. Cereb. Blood Flow Metab. 23, 1-18.

Ogawa, S., Lee, T.M., Nayak, A.S., Glynn, P., 1990. Oxygenation-sensitive contrast in magnetic resonance image of rodent brain at high magnetic fields. Magn. Reson. Med. 14, 68-78.

Okamoto, M., Dan, H., Sakamoto, K., Takeo, K., Shimizu, K., Kohno, S., Oda, I., Isobe, S., Suzuki, T., Kohyama, K., Dan, I., 2004. Threedimensional probabilistic anatomical cranio-cerebral correlation via the international 10-20 system oriented for transcranial functional brain mapping. NeuroImage 21, 99-111.

Owen, W.J., Borowsky, R., Sarty, G.E., 2004. FMRI of two measures of phonological processing in visual word recognition: Ecological validity matters. Brain Lang. 90, 40-46.

Pena, M., Maki, A., Kovacic, D., Dehaene-Lambertz, G., Koizumi, H., Bouquet, F., Mehler, J., 2003. Sounds and silence: an optical topography study of language recognition at birth. Proc. Natl. Acad. Sci. U. S. A. 100, 11702-11705.

Plichta, M.M., Heinzel, S., Ehlis, A.-C., Pauli, P., Fallgatter, A.J., 2007. Model-based analysis of rapid event-related functional near-infrared spectroscopy (NIRS) data: a parametric validation study. NeuroImage $35,625-634$

Prabhakaran, R., Blumstein, S.E., Myers, E.B., Hutchison, E., Britton, B., 2006. An event-related fMRI investigation of phonological-lexical competition. Neuropsychologia 44, 2209-2221.

Price, C., 2000. The anatomy of language: contributions from functional neuroimaging. J. Anat. 197, 335-359.

Pugh, K.R., Mencl, W., Shaywitz, B., Shaywitz, S., Fulbright, R., Constable, R., Skudlarski, P., Marchione, K., Jenner, A., Fletcher, J., Liberman, A., Shankweiler, D., Katz, L., Lacadie, C., Gore, J., 2000. The angular gyrus in developmental dyslexia: task-specific differences in functional connectivity within posterior cortex. Psychol. Sci. 11, 51-56.

Ridderinkhof, K.R., van den Wildenberg, W.P.M., Segalowitz, S.J., Carter, C.S., 2004. Neurocognitive mechanisms of cognitive control: The role of prefrontal cortex in action selection, response inhibition, performance monitoring, and reward-based learning. Brain Cogn. 56, 129-140.

Sankoh, A.J., Huque, M.F., Dubey, S.D., 1997. Some comments on frequently used multiple endpoint adjustment methods in clinical trials. Stat. Med. 16, 2529-2542.

Steinbrink, J., Villringer, A., Kempf, F., Haux, D., Boden, S., Obrig, H., 2006. Illuminating the BOLD signal: combined fMRI-fNIRS studies. Magn. Reson. Imaging 24, 495-505.

Schroeter, M.L., Bucheler, M.M., Muller, K., Uludag, K., Obrig, H., Lohmann, G., Tittgemeyer, M., Villringer, A., von Cramon, D.Y., 2004 Towards a standard analysis for functional near-infrared imaging. NeuroImage 21, 283-290.

Taga, G., Asakawa, K., Hirasawa, K., Konishi, Y., 2003. Hemodynamic responses to visual stimulation in occipital and frontal cortex of newborn infants: A near-infrared optical topography study. Early Hum. Dev. 75, S203-S210.

Thompson-Schill, S.L., D’Esposito, M., Aguirre, G., Farah, M., 1997. Role of left inferior prefrontal cortex in retrieval of semantic knowledge: A reevaluation. Proc. Natl. Acad. Sci. U. S. A. 94, 14792-14797.

Tsuzuki, D., Jurcak, V., Singh, A.K., Okamoto, M., Watanabe, E., Dan, I., 2007. Virtual spatial registration of stand-alone fNIRS data to MNI space. NeuroImage 34, 1506-1518.

Tzourio-Mazoyer, N., Landeau, B., Papathanassiou, D., Crivello, F., Etard, O., Delcroix, N., Mazoyer, B., Joliot, M., 2002. Automated anatomical labeling of activations in SPM using a macroscopic anatomical parcellation of the MNI MRI single-subject brain. NeuroImage 15, 273-289.

Villringer, A., Dirnagl, U., 1995. Coupling of brain activity and cerebral blood flow: basis of functional neuroimaging. Cerebrovasc. Brain Metab. Rev. 7, 240-276.

Wartenburger, I., Steinbrink, J., Telkemeyer, S., Friedrich, M., Friederici, A., Obrig, H., 2007. The processing of prosody: Evidence of interhemispheric specialization at the age of four. NeuroImage 34, 416-425.

Watanabe, E., Maki, A., Kawaguchi, F., Takashiro, K., Yamashita, Y., Koizumi, H., Mayanagi, Y., 1998. Non-invasive assessment of language dominance with near-infrared spectroscopic mapping. Neurosci. Lett. 256, 49-52.

Yeung, N., Botvinick, M.M., Cohen, J.D., 2004. The Neural Basis of Error Detection: Conflict Monitoring and the Error-Related Negativity. Psychol. Rev. 111, 931-959.

Zabel, T.A., Chute, D.L., 2002. Educational neuroimaging: A proposed neuropsychological application of near-infrared spectroscopy (nIRS). J. Head Trauma Rehabil. 17, 477-488. 